\title{
Les objets sous contrainte. Gages, saisies, confiscations, vols, pillages, recel au Moyen Âge
}

Auxerre, Centre d'études médiévales

Séminaire, 20-21 novembre 2009

Laurent Feller et Ana Rodriguez Lopez

\section{(2) OpenEdition}

\section{Journals}

Édition électronique

URL : https://journals.openedition.org/cem/11524

DOI : $10.4000 /$ cem. 11524

ISSN : 1954-3093

\section{Éditeur}

Centre d'études médiévales Saint-Germain d'Auxerre

Édition imprimée

Pagination : 169-175

ISSN : 1623-5770

\section{Référence électronique}

Laurent Feller et Ana Rodriguez Lopez, « Les objets sous contrainte. Gages, saisies, confiscations, vols,

pillages, recel au Moyen Âge », Bulletin du centre d'études médiévales d'Auxerre | BUCEMA [En ligne], 14 | 2010, mis en ligne le 14 octobre 2010, consulté le 22 septembre 2022. URL : http:// journals.openedition.org/cem/11524 ; DOI : https://doi.org/10.4000/cem.11524

\section{Ce document a été généré automatiquement le 22 septembre 2022}

Creative Commons - Attribution - Pas d'Utilisation Commerciale - Partage dans les Mêmes Conditions 4.0 International - CC BY-NC-SA 4.0

https://creativecommons.org/licenses/by-nc-sa/4.0/ 


\section{Les objets sous contrainte. Gages, saisies, confiscations, vols, pillages, recel au Moyen Âge}

Auxerre, Centre d'études médiévales

Séminaire, 20-21 novembre 2009

Laurent Feller et Ana Rodriguez Lopez

1 Du jeudi 20 au samedi 21 novembre 2009, à l'initiative d'Ana Rodriguez et de Laurent Feller, s'est tenu à Auxerre, dans la maison du Coche d'Eau, un séminaire d'études international portant sur Les objets sous contrainte. Gages, saisies, confiscation, vol, pillage, recel au Moyen Âge. Organisée conjointement par le Lamop, l'université de Paris 1 et le CCHS (CSIC) de Madrid, partiellement financée par un projet Picasso de l'association Égide, l'opération a bénéficié du soutien de l'université de Paris 1, du Framespa (université de Toulouse 2) et du CEM d'Auxerre.

2 Relevant du programme "La circulation des richesses au Moyen Âge», organisé et financé par le Lamop, ce séminaire faisait suite à une autre réunion tenue à Madrid en 2008, intitulée Circulation et remploi des objets au Moyen Âge. Dans l'un et l'autre cas, on désirait s'interroger sur les échanges d'objets en dehors des contextes marchands.

3 L'idée centrale du projet est que, dans le cadre de transactions effectuées en dehors du marché, des relations d'un type particulier se nouent entre les parties et que les objets en sont le support: pour comprendre exactement ce qui se passe entre les personnes qui traitent ensemble, il est apparu nécessaire de s'interroger à la fois sur les réutilisations des objets détournés de leur fonction première - ce fut le but du colloque de Madrid - et sur la nature de la circulation en dehors du marché. Sur la place d'échanges, les acteurs transigent: ils négocient, évaluent la qualité de l'objet, l'apprécient ou le déprécient, lui attribuent une valeur et définissent la contrepartie qui permettra de sceller l'échange et de le rendre profitable aux deux parties. Le prix résulte d'une négociation complexe, qui, intégrant différents paramètres comme l'utilité, la rareté ou la qualité, est le fruit de la liberté des acteurs. Dans le cas où l'on se situe en dehors du marché, l'objet peut circuler pour satisfaire un besoin précis, mais 
les modalités de son appréciation diffèrent, parce qu'il s'agit de produire d'autres effets que la satisfaction immédiate de ce besoin. D'autre part, et ce fut le sens de la réflexion menée à Madrid en 2008, les objets changent de fonction, d'utilité et de sens en passant de main en main dans des contextes où l'utilité n'est pas le seul but de l'échange. Par exemple, l'objet transmis lors de l'investiture d'une terre sert de support au droit exercé sur la terre et se dégage de sa fonction première. S'il s'agit d'une épée, celle-ci cesse d'être seulement une arme pour devenir un signe de la possession. Exhibée dans un procès, elle prouve l'existence de l'investiture, jusqu'au moment, où, la cérémonie fondatrice ayant été oubliée, elle redevient une arme et apparait comme une menace aux juges ayant à connaître l'affaire. S'interroger sur les objets revenait ainsi à se demander ce que les acteurs font effectivement lorsqu'ils échangent des objets contre d'autres objets et en transforment le statut. Des objets liés à production ou à la fonction peuvent ainsi devenir des objets précieux, voire, dans certaines circonstances, acquérir un statut sacré. Inversement, d'ailleurs, la transmission des objets ou leur échange contribue à assurer ou à transformer le statut des parties transactantes. C'est tout à fait clair dans le cas d'une investiture, où l'objet remis signifie le passage d'une personne d'une catégorie à une autre: la remise d'un anneau désigne le mariage, celui d'une crosse l'investiture épiscopale ou abbatiale. Bref, considérer l'échange non marchand et s'interroger sur la signification des objets employés et sur les transformations qu'ils subissent permet de s'interroger sur les liens entre les hommes, sur leur constitution et leur consolidation.

4 On était là dans une sphère où les hommes échangent librement, à l'intérieur simplement des contraintes sociales et des obligations posées par les règles de réciprocité dans les échanges. Or, si l'on considère les objets, c'est-à-dire si, d'une certaine façon, on prend le point de vue des choses, il existe des circonstances et des situations où de fortes contraintes externes existent et déterminent leur circulation. L'étude de ces circonstances et de ces situations était précisément le but de la rencontre d'octobre 2009.

Quatorze communications ont été présentées. Elles s'articulaient autour de trois thèmes distincts, les gages contractés ou cédés lors d'emprunts ou d'opérations de crédit; l'utilisation d'objets non monétaires lors des paiements; et, enfin, la guerre et le pillage.

6 La première section a permis de réfléchir aux conditions dans lesquelles les objets sont transférés d'un possesseur à un autre pour servir de couverture à des opérations de crédit et ce qu'il en résulte. Les objets servent alors de garantie, mais ne sont pas systématiquement immobilisés: les gages circulent, devenant alors des objets permettant de compenser d'autres échanges, parfois commerciaux, comme c'est le cas en Catalogne aux XIV et $\mathrm{XV}^{\mathrm{e}}$ siècles (C. Denjean). Les trocs qui sont documentés posent la question du juste prix d'une part et, d'autre part, apparaissent liés à des mécanismes d'extorsion, dans la mesure où l'une des parties, celle qui reçoit des objets, est contrainte d'en passer par là. Cela entraîne de nombreuses difficultés. Si on comprend bien la nécessité de l'opération dans le cas de pénurie monétaire, comme en Toscane au XII siècle (E. Huertas), il n'en va pas toujours de même. En Toscane, l'exemple d'un paiement où un crucifix de valeur circule de mains en mains pour permettre le paiement d'une rançon permet de montrer que l'objet, quoique précieux, a un prix clairement connu ou admis de tous. L'objet, ici, a circulé à la place de l'argent dans un contexte relativement neutre, le paiement d'une rançon en cas de guerre n'impliquant ni renforcement de l'hostilité ni établissement d'une relation positive d'amitié entre le 
prisonnier et son gardien. En revanche, à Marseille, au Xve siècle, la mise en circulation des objets peut se faire dans un fort cadre affectif (J. Sibon). Les relations entre juifs et chrétiens permettent de voir cela. Il n'y a aucun interdit sur les relations commerciales entre les deux communautés et, même, entre parties contractantes, des relations d'amitié peuvent exister: donner un livre en gage à un prêteur se fait et l'exemple développé, celui d'un juif engageant un livre auprès d'un chrétien, est fortement connoté en ce sens. Mais, prêté pour sa valeur commerciale, il est mis en circulation au moment où les relations se tendent entre l'emprunteur et son créancier, pour des raisons qui ne sont pas liées à la relation de crédit. La mise en circulation du livre, servant de couverture à une nouvelle opération de crédit, est très clairement un geste d'inimitié et de défiance qui amène les deux hommes à combattre à front renversé, le juif obtenant du tribunal ecclésiastique l'excommunication du chrétien pour nonremboursement de sa dette!

7 À Marseille, encore, D. L. Smail montre, par l'étude des inventaires, que la valeur des objets est connue. On investit dans les objets mais on les fait également circuler sous la forme de gage, dans une chaîne où l'objet prêté assume le rôle d'une monnaie fiduciaire, puisque l'on connaît son prix et que l'on se repose sur la bonne foi du créancier pour pouvoir le récupérer en cas de nécessité. Lors des faillites, cela pose de véritables problèmes qui se règlent par des saisies suivies de vente à l'encan, la saisie pouvant être suivie du rachat par le failli et ouvrant donc la voie à de nouvelles opérations de crédit. L'humiliation infligée par la procédure de saisie fait également partie du jeu de l'échange et peut être compensée par les procédures de rachat. Les objets, là aussi, exercent une fonction monétaire et la mise en gage est évidemment la forme choisie pour obtenir les liquidités nécessaires.

8 Certains objets, cependant, ne peuvent pas circuler, comme le rappelle F. Arias à propos de la Catalogne des XIII ${ }^{\mathrm{e}}$ et $\mathrm{XIV}^{\mathrm{e}}$ siècles, où il existe des prohibitions sur les ventes de chevaux, interdits à l'exportation et dont la circulation par le biais de l'échange marchand est réglementée pour des questions de statut social du possesseur: instrument de guerre et support de prestige, il ne peut être jeté sur le marché dans n'importe quelle condition. Le cheval est, par définition, un objet royal que seul le don permet de mettre en circulation de façon légitime, parce que les échanges à forte connotation symbolique le valorisent comme ils valorisent aussi les personnes qui en bénéficient.

9 La valorisation des objets s'effectue de toute sorte de manières. Le marché de l'occasion en est une essentielle dans tout l'Occident (J.Marsilla). Étudié plus spécifiquement pour Valence, il permet d'intégrer les notions d'usure et de vieillissement dans l'estimation de la valeur. Le marché des biens d'occasion est extrêmement actif et on y a recours pour toute sorte de raison, notamment lors de la liquidation des successions. La question de la place que prennent les objets volés sur un tel marché est évidemment importante mais ne peut être réglée aisément. Concernant pour beaucoup les vêtements, ce marché permet de recycler des objets passés de mode. Il permet aussi de se procurer des matières premières, le linge usagé pouvant être retransformé en d'autres produits. Il suppose de toute façon une grande capacité à identifier et à évaluer de la part de qui veut y être actif. Enfin, ce système, encore une fois, sert à obtenir des liquidités en mobilisant des objets provenant de l'environnement domestique, mais qui peuvent avoir été acquis précisément pour servir de réserve de valeur. 
10 J. Claustre pose également, dans le cadre de la France du $\mathrm{xv}^{\mathrm{e}}$ siècle, la question de l'accès à la liquidité et insiste sur le fait que l'on fait davantage circuler les objets que le numéraire. Acheter sur gage permet de différer les paiements et acheter des objets permet de ne pas épargner sous une forme liquide, le gage mobilier étant dans ces conditions une forme particulière de liquidité.

11 Les objets, y compris semi-finis, peuvent servir directement de moyen de paiement, en dehors même de toute forme de prise de gage ou de mise en circulation des gages. C. Verna montre que le fer, sous forme de lingots ou de barres, peut être utilisé comme moyen de paiement des ouvriers qui le fabriquent, du moins dans le contexte de la vallée du Trets au $\mathrm{xv}^{\mathrm{e}}$ siècle. On y évalue normalement en monnaie, mais on paie fréquemment en fer, et ce que l'on paie ainsi ce sont les salaires et les amendes. Le paiement des salaires en fer touche des catégories très particulières de travailleurs migrants, hautement spécialisés, en l'occurrence les Ariégeois et les Basques, sur lesquels pèsent des contraintes sociales particulières, qui, d'une part, permettent à leurs employeurs de les payer de la sorte et qui, d'autre part, leur sont utiles. Le système repose sur une évaluation précise de la qualité du fer en jeu, connue par référence au nom de l'atelier qui l'a produit.

12 Les hôtels royaux ou princiers sont de grands réservoirs d'objets. L'organisation de la vie quotidienne des rois ou des princes, ainsi que l'aspect cérémoniel qu'y prend toute chose, implique de détenir des objets en grand nombre et de toute sorte. Cette présence massive d'objets de valeur concentrés en un même endroit implique des procédures de surveillance, des vols ou des formes de réappropriation des objets se produisant normalement dans la domesticité, quel que soit le niveau de celle-ci. Les responsables de l'hôtel doivent donc à la fois gérer des choses et contrôler des personnes à travers ces procédures de gestion, la tentation étant grande de se servir et de revendre. La surveillance des objets et des procédures apparait donc comme un moyen de contrôler les hommes et les femmes travaillant dans l'hôtel et de contribuer ainsi à l'affirmation de l'autorité royale sur des lieux et sur des personnes dont dépend la mise en scène de la majesté.

13 À travers une réflexion sur le don, A. Wilkin propose, pour sa part, une étude sur les circulations ritualisées durant le haut Moyen Âge. Le pillage est ainsi suivi de redistributions effectuées après les opérations militaires. Les objets sacrés sont également des réservoirs de valeur et peuvent être «désacralisés» à tout instant. Ces utiles rappels servent de transition avec la réflexion menée par R. Keller sur l'économie du pillage aux frontières de l'Empire carolingien puis ottonien aux $\mathrm{IX}^{\mathrm{e}}$ et $\mathrm{x}^{\mathrm{e}}$ siècles. La frontière, lieu du conflit, est aussi celui de l'échange, du fait des pillages et des partages qui le suivent. Des règles strictes l'organisent: il faut à la fois répartir entre les différents ayants droit, les guerriers, mais aussi redistribuer. C'est l'occasion pour le chef de guerre de montrer son autorité et sa largesse, les deux logiques, celle du partage et celle de la redistribution, pouvant être (et étant parfois) antagonistes.

14 Le pillage donne lieu à une véritable économie, comme le montre V. Toureille en étudiant les pratiques des écorcheurs en Bourgogne au Xve siècle. Les pillages et les rançons peuvent y apparaître comme une forme particulièrement brutale et non normée du prélèvement seigneurial sur les biens, la production et les personnes. Tous les moyens sont bons pour se procurer des objets: la destruction «pour l'exemple», l'intimidation, le pillage pur et simple. La valeur des objets est connue parce qu'il y a eu une enquête entre 1440 et 1444 et que, dans celle-ci, les plaignants ont dit quelles 
étaient les valeurs des objets qui leur avaient été dérobés. L'estimation est ponctuelle et son rapport avec la valeur réelle des objets, dans le cadre d'un échange monétaire, problématique. Cela dit, la mise aux enchères du butin vendu donne une idée des montants. Les partages se font ensuite en fonction du grade. Les informateurs sont rétribués. Bref, il y a des procédures qui permettent de mesurer la valeur.

15 J. J. Larrea aborde la question du destin des objets pillés dans l'Espagne chrétienne des $\mathrm{x}^{\mathrm{e}}$ et $\mathrm{XI}^{\mathrm{e}}$ siècles. Il montre, en illustrant son propos par des textes, que le butin fait l'objet de pratiques particulières. Il donne lieu à une consommation collective et immédiate, notamment s'agissant du vin, dans un cadre festif mais ritualisé. S'effectuant dans le cadre de vengeances, il fait partie d'un système de contrôle de la procédure vindicatoire, qui, collective, implique aussi des destructions.

16 Enfin, P. Sopena décrit la circulation des objets en temps de guerre dans le Léon des $\mathrm{XII}^{\mathrm{e}}-\mathrm{XIV}{ }^{\mathrm{e}}$ siècles. On pille, bien sûr. Mais on rachète également des captifs. La guerre est cependant une expérience complexe qui implique aussi le contact avec le sacrilège: les objets intéressant le pillard sont souvent des reliquaires ou des vases sacrés. Des réquisitions honorables, faites par le roi, qui sont en elles-mêmes fort proches d'un prélèvement brutal et non normé, d'un pillage pur et simple. L'obtention de liquidités se fait aussi, du point de vue du roi, dans le cadre d'échanges: cédant des terres et des droits, des privilèges fiscaux, par exemple, le roi obtient en échange les liquidités dont il a besoin sous la forme de monnaie, mais aussi de chevaux et d'ornements d'Église. L'équivalent en argent est connu, mais on ne peut véritablement parler, dans ce cas, d'échange marchand, dans la mesure où la contrainte de la guerre et celle de l'État sont évidentes. La nécessité dans laquelle se trouve le souverain d'avoir à trouver des espèces l'amène à extraire par la force des trésors d'Église. En 1116, la reine organise une véritable tournée de prédation dans ses États pour parvenir à financer la guerre. Ces prélèvements forcés sont aussi un instrument politique dans la mesure où ils permettent au roi d'exercer sa vengeance...

17 Les points mis en avant par la rencontre sont nombreux. On a relevé avec intérêt l'existence normale de moyens de paiements alternatifs. Les objets exercent clairement des fonctions monétaires, puisqu'ils circulent à la place de la monnaie. Dès l'instant qu'ils ont été institués en gage dans le cadre d'opérations financières, qui ne sont pas nécessairement des opérations de crédit, ils circulent librement en raison d'une valeur vénale que tous connaissent. Cet aspect de notre discussion amène naturellement à poser la question du troc et, de façon plus générale, des moyens de paiement non monétaires. Les acteurs y recourent parfois, mais pas toujours: les circonstances de ce recours devront faire l'objet d'une enquête.

18 D'autre part, le lien établi entre les personnes manipulant des choses a été souligné, notamment dans le cadre de prêts où de fortes implications affectives sont manifestes. Ce lien, alors horizontal, peut aussi être vertical: la redistribution des objets pillés est l'occasion pour le souverain de s'affirmer comme tel. De même, le pillage ou la réquisition forcée opérée sur ses propres terres peut être l'occasion pour le roi d'exercer une vengeance.

19 Enfin, une question demeure, celle de savoir comment et par quelles procédures la valeur des choses est mesurée. On entrevoit bien le fonctionnement du marché, à condition que l'on y ait recours. On voit aussi qu'il existe des agents spécialisés dans ces questions d'évaluation et c'est pourquoi nous continuerons ce programme en réfléchissant à l'expertise. 


\section{Rappel du programme}

- Fernando ARIAS, Cuando se intenta que los objetos se guarden. Los límites a la circulación de caballos en Castilla durante la primera mitad del siglo XIV

- Gil BARTholeyns, L'administration des objets dans la maison du roi: l'hôtel français sous surveillance, 1261-1323

- Julie CLAUSTRE, Objets gagés, objets saisis, objets vendus par la justice à Paris ( $\mathrm{XIV}^{\mathrm{e}}-\mathrm{XV}^{\mathrm{e}}$ siècles)

- Claude DENJEAN, Une autre façon de prêter: gages, saisies, extorsions en Catalogne, XIII ${ }^{\mathrm{e}-}$ XIV ${ }^{\mathrm{e}}$ siècles

- Juan Vicente garcía MARSILLA, Con las ropas de otro. El mercado del textil de segunda mano en la Valencia medieval

- Emmanuel HUERTAS, Recherches autour d'un crucifix: la mise en gage d'objets liturgiques en Toscane au XII ${ }^{\mathrm{e}}$ siècle

- Rodolphe KELLER, Partage, distribution et utilisation du butin dans le monde franc ( $\mathrm{VI}^{\mathrm{e}}$ IX ${ }^{\mathrm{e}}$ siècle)

- Juanjo LARREA, Le destin du butin dans les communautés de l'Espagne chrétienne autour du $\mathrm{XI}^{\mathrm{e}}$ siècle, entre le gaspillage et la remise au marché

- Pascual MARTíNEZ SOPENA, La circulation d'objets en temps de guerre. Les années 1110-1130 à Léon

- Juliette SIBON, Du gage-objet au gage-chose: une étude de cas au sommet de la société urbaine marseillaise à l'extrême fin du XIV siècle

- Daniel L. SMAïL, Goods as Hostages. Aspects of the Process of Debt Recovery in Marseille and Lucca in the Later Middle Ages

- Valérie toureILle, L'économie du pillage. L'exemple des écorcheurs à la fin de la guerre de Cent Ans

- Catherine VERNA, Payer avec du fer et contrôler les migrants: un marché sous contraintes (Vallespir, $\mathrm{XV}^{\mathrm{e}}$ siècle)

- Alexis WILKIN, La circulation contrainte des objets au haut Moyen Âge, entre coercition «institutionnalisée» et pillage

INDEX

Mots-clés : gage, saisie, confiscation, vol, pillage 